\title{
KP Trigonometric Solitons and an Adelic Flag Manifold ${ }^{\star}$
}

\author{
Luc HAINE
}

Department of Mathematics, Université catholique de Louvain, Chemin du Cyclotron 2, 1348 Louvain-la-Neuve, Belgium

E-mail: haine@math.ucl.ac.be

Received November 22, 2006, in final form January 5, 2007; Published online January 27, 2007

Original article is available at http://www.emis.de/journals/SIGMA/2007/015/

\begin{abstract}
We show that the trigonometric solitons of the KP hierarchy enjoy a differentialdifference bispectral property, which becomes transparent when translated on two suitable spaces of pairs of matrices satisfying certain rank one conditions. The result can be seen as a non-self-dual illustration of Wilson's fundamental idea [Invent. Math. 133 (1998), 1-41] for understanding the (self-dual) bispectral property of the rational solutions of the KP hierarchy. It also gives a bispectral interpretation of a (dynamical) duality between the hyperbolic Calogero-Moser system and the rational Ruijsenaars-Schneider system, which was first observed by Ruijsenaars [Comm. Math. Phys. 115 (1988), 127-165].
\end{abstract}

Key words: Calogero-Moser type systems; bispectral problems

2000 Mathematics Subject Classification: 35Q53; 37K10

Dedicated to the memory of Vadim Kuznetsov

\section{Introduction}

One of the many gems I had the chance to share with Vadim Kuznetsov during his visit to Louvain-la-Neuve in the fall semester of 2000, had to do with his work on separation of variables and spectrality. He knew about my work on bispectral problems and insisted that these problems were connected. He introduced me to his work with Nijhoff and Sklyanin [11], on separation of variables for the elliptic Calogero-Moser system. I would have loved to discuss the topic of the present paper with him, which deals with the (simpler) trigonometric version of this system.

One of the exciting new developments in the field of integrable systems has been the introduction by George Wilson [21] of the so called Calogero-Moser spaces

$$
C_{N}=\{(X, Z) \in g l(N, \mathbb{C}) \times g l(N, \mathbb{C}): \operatorname{rank}([X, Z]+I)=1\} / G L(N, \mathbb{C}),
$$

where $g l(N, \mathbb{C})$ denotes the space of complex $N \times N$ matrices, $I$ is the identity matrix and the complex linear group $G L(N, \mathbb{C})$ acts by simultaneous conjugation of $X$ and $Z$. These spaces are at the crossroads of many areas in mathematics, connecting with such fields as non-commutative algebraic and symplectic geometry. For an introduction as well as a broad overview of the subject, I recommend Etingof's recent lectures [4] at ETH (Zürich). Since the bispectral problem is not mentioned in these lectures, it seems not inappropriate to start from this problem as introduced in the seminal paper [3] by Duistermaat and Grünbaum, which has played and (as we shall see) continues to play a decisive role in the subject.

*This paper is a contribution to the Vadim Kuznetsov Memorial Issue "Integrable Systems and Related Topics". The full collection is available at http://www.emis.de/journals/SIGMA/kuznetsov.html 
In the form discussed by Wilson [20], the bispectral problem asks for the classification of all rank 1 commutative algebras $\mathcal{A}$ of differential operators, for which the joint eigenfunction $\psi(x, z)$ which satisfies

$$
A(x, \partial / \partial x) \psi(x, z)=f_{A}(z) \psi(x, z) \quad \forall A \in \mathcal{A},
$$

also satisfies a (non-trivial) differential equation in the spectral variable

$$
B(z, \partial / \partial z) \psi(x, z)=g(x) \psi(x, z) .
$$

In [20], it was found that all the solutions of the problem are parametrized by a certain subgrassmannian of the Segal-Wilson Grassmannian Gr, that Wilson called the adelic Grassmannian and that he denoted by $\mathrm{Gr}^{\text {ad }}$. The same Grassmannian parametrizes the rational solutions in $x$ of the Kadomtsev-Petviashvili (KP) equation (vanishing as $x \rightarrow \infty$ ). The main result of [21] is to give another description of $\mathrm{Gr}^{\text {ad }}$ as the union $\cup_{N \geq 0} C_{N}$ of the Calogero-Moser spaces introduced above. The correspondence can be seen as given by the map

$$
\beta:(X, Z) \rightarrow \psi_{W}(x, z)=e^{x z} \operatorname{det}\left\{I-(x I-X)^{-1}(z I-Z)^{-1}\right\},
$$

which sends a pair $(X, Z) \in C_{N}$ (modulo conjugation) to the (stationary) Baker-Akhiezer function of the corresponding space $W=\beta(X, Z) \in \mathrm{Gr}^{\text {ad }}$. From (1.4), one sees immediately that the mysterious bispectral involution $b: \mathrm{Gr}^{\text {ad }} \rightarrow \mathrm{Gr}^{\text {ad }}$ which exchanges the role of the variables $x$ and $z$

$$
\psi_{b(W)}(x, z)=\psi_{W}(z, x), \quad W \in \mathrm{Gr}^{\mathrm{ad}},
$$

becomes transparent when expressed at the level of the Calogero-Moser spaces, as it is given by $b^{C}(X, Z)=\left(Z^{t}, X^{t}\right)$, where $X^{t}$ and $Z^{t}$ are the transposes of $X$ and $Z$. The situation is nicely summarized by the following commutative diagram, all arrows of which are bijections

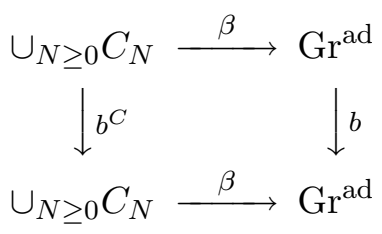

In [6], jointly with Plamen Iliev, we considered the following discrete-continuous version of the bispectral problem. To determine all rank 1 commutative algebras $\mathcal{A}$ of difference operators, for which the joint eigenfunction $\psi(n, z)$ which satisfies

$$
A \psi(n, z) \equiv \sum_{\text {finitely many } j \in \mathbb{Z}} a_{j}(n) \psi(n+j, z)=f_{A}(z) \psi(n, z) \quad \forall A \in \mathcal{A},
$$

also satisfies a (non-trivial) differential equation in the spectral variable

$$
B(z, \partial / \partial z) \psi(n, z)=g(n) \psi(n, z) .
$$

The problem was motivated by an earlier work with Alberto Grünbaum [5], where we investigated the situation when the algebra $\mathcal{A}$ contains a second-order symmetric difference operator (this time, without imposing any rank condition on $\mathcal{A}$ ). This situation extends the theory of the classical orthogonal polynomials, where the differential equation is of the second order too.

The main result of [6] was to construct from Wilson's adelic Grassmannian Grad an (isomorphic) adelic flag manifold $\mathrm{Fl}^{\text {ad }}$, which provides solutions of the bispectral problem raised above, and parametrizes rational solutions in $n$ (vanishing as $n \rightarrow \infty$ ) of the discrete KP hierarchy. 
The message of this paper is to show that the analogue of Wilson's diagram (1.5) in the context of this new bispectral problem is the following commutative diagram, with bijective arrows

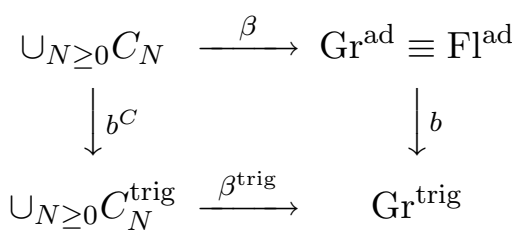

In this diagram, the spaces

$$
C_{N}^{\text {trig }}=\left\{(X, Z) \in G L(N, \mathbb{C}) \times g l(N, \mathbb{C}): \operatorname{rank}\left(X Z X^{-1}-Z+I\right)=1\right\} / G L(N, \mathbb{C}),
$$

are trigonometric analogues of the Calogero-Moser spaces $C_{N}$ defined in (1.1). Gr ${ }^{\text {trig }}$ is a certain subgrassmannian of linear spaces $W \in$ Gr parametrizing special solitons of the KP hierarchy, that I call the "trigonometric Grassmannian", since the corresponding tau functions $\tau_{W}$ take the form

$$
\tau_{W}\left(x+t_{1}, t_{2}, t_{3}, \ldots\right)=\prod_{i=1}^{N} 2 \sinh \frac{\left(x-x_{i}\left(t_{1}, t_{2}, t_{3}, \ldots\right)\right)}{2},
$$

with $x_{i}\left(t_{1}, t_{2}, t_{3}, \ldots\right)$ being a solution of the trigonometric Calogero-Moser-Sutherland hierarchy (as long as all $x_{i}\left(t_{1}, t_{2}, t_{3}, \ldots\right)$ remain distinct, see Section 3 ). The bispectral map $b$ : $\mathrm{Fl}^{\text {ad }} \rightarrow$ $\mathrm{Gr}^{\text {trig }}$, which sends a flag $\mathcal{V}$ to a linear space $W=b(\mathcal{V})$, and is defined by

$$
\psi_{b(\mathcal{V})}(x, z)=\psi_{\mathcal{V}}\left(z, e^{x}-1\right)
$$

trivializes when expressed at the level of the Calogero-Moser spaces, as it is now given by ${ }^{1}$

$$
b^{C}(X, Z)=\left(I+Z^{t}, X^{t}\left(I+Z^{t}\right)\right) .
$$

The result will follow easily from the expression of the (stationary) Baker-Akhiezer function $\psi_{W}(x, z)$ of a space $W \in \mathrm{Gr}^{\text {trig }}$, in terms of pairs of matrices $(X, Z) \in C_{N}^{\text {trig }}$

$$
\psi_{W}(x, z)=e^{x z} \operatorname{det}\left\{I-X\left(e^{x} I-X\right)^{-1}(z I-Z)^{-1}\right\},
$$

which defines the map $\beta^{\text {trig }}$ in the diagram (1.8). The definition of the map $\beta$ in the same diagram follows immediately from the definition of the adelic flag manifold in terms of Wilson's adelic Grassmannian as given in [6], and will be recalled in Section 4.

Some time ago, Ruijsenaars [16] (see also [17]) made a thorough study of the action-angle maps for Calogero-Moser type systems with repulsive potentials, via the study of their scattering theory. Along the way, he observed various duality relations between these systems. In particular, when the interaction between the particles in the trigonometric Calogero-Moser system is repulsive, the system is dual (in the sense of scattering theory) to the rational RuijsenaarsSchneider system. In the last section, we show that within our picture $(1.8)$, if $\tau_{W}\left(t_{1}, t_{2}, t_{3}, \ldots\right)$ is the tau function of a space $W \in \mathrm{Gr}^{\text {trig }}$ as in (1.10), the tau function of the flag $b^{-1}(W)$ is

$$
\tau_{b^{-1}(W)}\left(n, t_{1}, t_{2}, \ldots\right)=\prod_{i=1}^{N}\left(n-\lambda_{i}\left(t_{1}, t_{2}, \ldots\right)\right), \quad n \in \mathbb{Z},
$$

with $\lambda_{i}\left(t_{1}, t_{2}, \ldots\right)$ solving now the rational Ruijsenaars-Schneider hierarchy, thus representing Ruijsenaars' duality as a bispectral map. The likelihood of this last statement was formulated previously by Kasman [8], on the basis of a similar relationship between the quantum versions of these systems, as studied by Chalykh [1] (see also [2]). It is also implicitly suggested by a recent work of Iliev [7], which relates the polynomial tau functions in $n$ of the discrete KP hierarchy with the rational Ruijsenaars-Schneider hierarchy.

\footnotetext{
${ }^{1}$ The additional condition $\operatorname{det}(I+Z) \neq 0$ needed for this definition to make sense, follows from fixing the radius of the circle used in the definition of Gr to be 1, which can always be assumed, see Section 4 .
} 


\section{Trigonometric solitons of the KP hierarchy}

In this section, we construct a class of special solitons of the KP hierarchy. Their relation with the trigonometric version of the Calogero-Moser hierarchy will be explained in the next section, justifying the appellation "trigonometric solitons". We first need to recall briefly the definition of the Segal-Wilson Grassmannian Gr and its subgrassmannian Gr ${ }^{\text {rat }}$, from which solitonic solutions of the KP hierarchy can be constructed, see [18] for details. Let $S^{1} \subset \mathbb{C}$ be the unit circle, with center the origin, and let $H$ denote the Hilbert space $L^{2}\left(S^{1}, \mathbb{C}\right)$. We split $H$ as the orthogonal direct sum $H=H_{+} \oplus H_{-}$, where $H_{+}$(resp. $H_{-}$) consists of the functions whose Fourier series involves only non-negative (resp. only negative) powers of $z$. Then $\mathrm{Gr}$ is the Grassmannian of all closed subspaces $W$ of $H$ such that (i) the projection $W \rightarrow H_{+}$ is a Fredholm operator of index zero (hence generically an isomorphism); (ii) the projection $W \rightarrow H_{-}$is a compact operator. For $t=\left(t_{1}, t_{2}, \ldots\right)$, let $\exp (t, z)=\exp \left(\sum_{k=1}^{\infty} t_{k} z^{k}\right)$. For all $t$, $\exp ^{-1}(t, z) W$ belongs to $\mathrm{Gr}$ and, for almost any $t$, it is isomorphic to $H_{+}$, so that there is a unique function in it $\tilde{\psi}_{W}(t, z)$ which projects onto 1 . The function $\psi_{W}(t, z)=\exp (t, z) \tilde{\psi}_{W}(t, z)$ is called the Baker-Akhiezer function of the space $W$ and $\tilde{\psi}_{W}(t, z)$ is called the reduced Baker-Akhiezer function. A fundamental result of Sato asserts that there is a unique (up to multiplication by a constant) function $\tau_{W}\left(t_{1}, t_{2}, t_{3}, \ldots\right)$, the celebrated tau function, such that

$$
\psi_{W}(t, z)=\exp (t, z) \frac{\tau_{W}\left(t_{1}-1 / z, t_{2}-1 /\left(2 z^{2}\right), t_{3}-1 /\left(3 z^{3}\right), \ldots\right)}{\tau_{W}\left(t_{1}, t_{2}, t_{3}, \ldots\right)} .
$$

An element of the form $\sum_{k \leq s} a_{k} z^{k}, a_{s} \neq 0$, is called an element of finite order $s$. For $W \in \mathrm{Gr}$, $W^{\text {alg }}$ denotes the subspace of elements of finite order of $W$. It is a dense subspace of $W$. We also need the ring

$$
A_{W}=\left\{f \text { analytic in a neighborhood of } S^{1}: f . W^{\text {alg }} \subset W^{\text {alg }}\right\} .
$$

The rational Grassmannian $\mathrm{Gr}^{\mathrm{rat}}$ is the subset of $\mathrm{Gr}$, for which $\operatorname{Spec}\left(A_{W}\right)$ is a rational curve. In this case, $A_{W}$ is a subset of the ring $\mathbb{C}[z]$ of polynomials in $z$ and the map $\mathbb{C} \rightarrow \operatorname{Spec}\left(A_{W}\right)$ induced by the inclusion is a birational isomorphism, sending $\infty$ to a smooth point completing the curve.

Let us fix $N$ distinct complex numbers $\lambda_{1}, \ldots, \lambda_{N}$ inside of $S^{1}$, and another $N$ non-zero complex numbers $\mu_{1}, \ldots, \mu_{N}$. We assume that $\lambda_{i}-\lambda_{j} \neq 1, \forall i \neq j$. We define $W_{\lambda, \mu}^{\text {alg }}$ as the space of rational functions $f(z)$ such that

(i) $f$ is regular except for (at most) simple poles at $\lambda_{1}, \ldots, \lambda_{N}$ and poles of any order at infinity;

(ii) $f$ satisfies the $N$ conditions

$$
\operatorname{res}_{\lambda_{i}} f(z)+\mu_{i}^{2} f\left(\lambda_{i}-1\right)=0, \quad 1 \leq i \leq N,
$$

where $\operatorname{res}_{\lambda_{i}} f(z)$ is the residue of $f(z)$ at $\lambda_{i}$. The closure of $W_{\lambda, \mu}^{\text {alg }}$ in $L^{2}\left(S^{1}, \mathbb{C}\right)$ defines a space $W_{\lambda, \mu} \in \mathrm{Gr}^{\mathrm{rat}}$.

The Baker-Akhiezer function $\psi_{W}$ of a space $W=W_{\lambda, \mu}$ has the form

$$
\psi_{W}(t, z)=\exp (t, z)\left\{1-\sum_{j=1}^{N} \frac{\mu_{j} b_{j}(t)}{z-\lambda_{j}}\right\},
$$

for some functions $b_{j}(t)$ determined by (2.3). By a simple computation, we obtain

$$
-\exp \left(t, \lambda_{i}\right) b_{i}(t)+\mu_{i} \exp \left(t, \lambda_{i}-1\right)\left\{1+\sum_{j=1}^{N} \frac{\mu_{j} b_{j}(t)}{1+\lambda_{j}-\lambda_{i}}\right\}=0 .
$$


We introduce the $N \times N$ matrices $X$ and $Z$ with entries

$$
X_{i j}=\frac{\mu_{i} \mu_{j}}{1+\lambda_{j}-\lambda_{i}}, \quad Z_{i j}=\lambda_{i} \delta_{i j},
$$

with $\delta_{i j}$ the usual Kronecker symbol so that $Z$ is diagonal, and we put

$$
\tilde{X}=\exp \left\{\sum_{k=1}^{\infty} t_{k}\left(Z^{k}-(Z-I)^{k}\right)\right\}-X .
$$

With these notations, the system of equations (2.5) determining the functions $b_{i}(t)$ is written as

$$
\tilde{X} b(t)=\mu
$$

where $b(t)$ and $\mu$ denote column vectors of length $N$, with entries $b_{i}(t)$ and $\mu_{i}$ respectively. From (2.4) and (2.8), we get that the reduced Baker-Akhiezer function $\tilde{\psi}_{W}(t, z)=\exp ^{-1}(t, z) \psi_{W}(t, z)$ can be written as

$$
\tilde{\psi}_{W}(t, z)=1-\mu^{t}(z I-Z)^{-1} \tilde{X}^{-1} \mu,
$$

with $\mu^{t}$ the row vector obtained by transposing $\mu$.

The following commutation relation will be crucial

$$
[X, Z]=\mu \mu^{t}-X
$$

For short set

$$
\tilde{Z}=z I-Z \text {. }
$$

Considering that if $T$ is a matrix of rank 1 then $1+\operatorname{tr} T=\operatorname{det}(I+T)$ (with $\operatorname{tr}$ denoting the trace), from (2.7), (2.9), (2.10) and (2.11), we find

$$
\begin{aligned}
\tilde{\psi}_{W}(t, z) & =1-\operatorname{tr}\left\{\tilde{X}^{-1} \mu \mu^{t} \tilde{Z}^{-1}\right\}=\operatorname{det}\left\{I-\tilde{X}^{-1}([X, Z]+X) \tilde{Z}^{-1}\right\} \\
& =\operatorname{det}\left\{I-\tilde{X}^{-1}([\tilde{X}, \tilde{Z}]+X) \tilde{Z}^{-1}\right\}=\operatorname{det}\left\{\tilde{X}^{-1}(\tilde{Z} \tilde{X}-X) \tilde{Z}^{-1}\right\} .
\end{aligned}
$$

Using the fact that the determinant of a product of matrices does not depend on the orders of the factors, we get

$$
\tilde{\psi}_{W}(t, z)=\operatorname{det}\left\{I-X \tilde{X}^{-1} \tilde{Z}^{-1}\right\} .
$$

In particular, setting $t_{2}=t_{3}=\cdots=0$ and $t_{1}=x$, we find that the so-called stationary Baker-Akhiezer function of $W$ admits the following form

$$
\psi_{W}(x, z)=e^{x z} \operatorname{det}\left\{I-X\left(e^{x} I-X\right)^{-1}(z I-Z)^{-1}\right\} .
$$

By Cauchy's determinant formula applied to $X$ in (2.6)

$$
\operatorname{det}(X)=\prod_{i=1}^{N} \mu_{i}^{2} \prod_{1 \leq i<j \leq N}\left(1-\frac{1}{1-\left(\lambda_{i}-\lambda_{j}\right)^{2}}\right),
$$

which is non-zero by the assumptions we made on the $\lambda_{j}$ 's and $\mu_{j}$ 's. Thus $X$ is invertible and it follows from (2.10) that $\operatorname{rank}\left(X Z X^{-1}-Z+I\right)=1$, as announced in the Introduction, see (1.9) and (1.13). It is now easy to deduce the following proposition. 
Proposition 1. The tau function $\tau_{W}\left(t_{1}, t_{2}, t_{3}, \ldots\right)$ of a space $W=W_{\lambda, \mu}$ is given by

$$
\tau_{W}\left(t_{1}, t_{2}, t_{3}, \ldots\right)=\operatorname{det}\left\{I-X \exp \left\{\sum_{k=1}^{\infty} t_{k}\left((Z-I)^{k}-Z^{k}\right)\right\}\right\},
$$

with $X$ and $Z$ defined as in (2.6).

Proof. Denoting for short by $\exp \{\cdots\}$ the expression that appears inside the exponential in (2.15), one computes

$$
\begin{aligned}
& \tau_{W}\left(t_{1}-\frac{1}{z}, t_{2}-\frac{1}{2 z^{2}}, t_{3}-\frac{1}{3 z^{3}}, \cdots\right)=\operatorname{det}\left\{I-X \exp \{\cdots\}(z I-(Z-I))(z I-Z)^{-1}\right\} \\
& \quad=\operatorname{det}\left\{I-X \exp \{\cdots\}-X \exp \{\cdots\}(z I-Z)^{-1}\right\}
\end{aligned}
$$

from which it follows that

$$
\begin{aligned}
& \frac{\tau_{W}\left(t_{1}-1 / z, t_{2}-1 /\left(2 z^{2}\right), t_{3}-1 /\left(3 z^{3}\right), \ldots\right)}{\tau_{W}\left(t_{1}, t_{2}, t_{3}, \ldots\right)}=\operatorname{det}\left\{I-X\left(\exp ^{-1}\{\ldots\}-X\right)^{-1}(z I-Z)^{-1}\right\} \\
& \quad=\operatorname{det}\left\{I-X \tilde{X}^{-1} \tilde{Z}^{-1}\right\}
\end{aligned}
$$

with $\tilde{X}$ and $\tilde{Z}$ defined as in (2.7) and (2.11). This shows that the reduced Baker-Akhiezer function obtained in (2.12) satisfies Sato's formula (2.1) with $\tau_{W}$ as in (2.15). Since this formula determines the tau function up to a constant, the proof is complete.

Remark 1. ${ }^{2}$ Kasman and Gekhtman [9] (see Corollary 3.2 in their paper) have established that for any triple $(X, Y, Z)$ of $N \times N$ matrices such that rank $(X Z-Y X)=1$, the function

$$
\tau_{(X, Y, Z)}\left(t_{1}, t_{2}, \ldots\right)=\operatorname{det}\left\{I-X \exp \left\{-\sum_{k=1}^{\infty} t_{k} Z^{k}\right\} \exp \left\{\sum_{k=1}^{\infty} t_{k} Y^{k}\right\}\right\}
$$

is a tau function of the KP hierarchy, associated with some $W \in \mathrm{Gr}^{\mathrm{rat}}$, by showing that it satisfies the Hirota equation in Miwa form. The special choice

$$
X_{i j}=\frac{\mu_{i} \mu_{j}}{\lambda_{j}-\nu_{i}}, \quad Y_{i j}=\nu_{i} \delta_{i j}, \quad Z_{i j}=\lambda_{i} \delta_{i j}, \quad \text { with } \quad \nu_{i} \neq \lambda_{j}, \quad \forall i, j,
$$

leads to a $N$-soliton solution of the KP hierarchy, and Proposition 1 can be obtained by picking $Y=Z-I$.

Thanks to Remark 1, it makes sense to introduce the following definition:

Definition 1. The trigonometric Grassmannian $\mathrm{Gr}^{\text {trig }}$ is defined to be the following subgrassmannian of $\mathrm{Gr}^{\text {rat }}$

$$
\mathrm{Gr}^{\mathrm{trig}}=\left\{W \in \mathrm{Gr}^{\mathrm{rat}}: \tau_{W}\left(t_{1}, t_{2}, \ldots\right)=\tau_{(X, Z-I, Z)}\left(t_{1}, t_{2}, \ldots\right), \text { for }(X, Z) \in \bigcup_{N \geq 0} C_{N}^{\text {trig }}\right\}
$$

with $C_{N}^{\text {trig }}$ defined as in (1.9) and with $\tau_{(X, Z-I, Z)}\left(t_{1}, t_{2}, \ldots\right)$ defined as in (2.16). The corresponding solutions of the KP hierarchy will be called trigonometric solitons.

\footnotetext{
${ }^{2}$ I am thankful to the two referees both of whom made this important observation.
} 
Example 1. As an example of a non-generic $W \in \mathrm{Gr}^{\text {trig }}$ (i.e. not of the form $W_{\lambda, \mu}$ as above), let us define $W \in \mathrm{Gr}^{\text {rat }}$ to be the closure in $L^{2}\left(S^{1}, \mathbb{C}\right)$ of the space $W^{\text {alg }}$ formed with the rational functions $f(z)$ such that

(i) $f$ is regular except for (at most) a double pole at $z=0$ and a pole of any order at $\infty$;

(ii) $f$ satisfies the two conditions

$$
\begin{aligned}
& \operatorname{res}_{0} z f(z)+f(-1)=0, \\
& \operatorname{res}_{0} f(z)+f^{\prime}(-1)=0 .
\end{aligned}
$$

A simple computation shows that the corresponding stationary Baker-Akhiezer function is

$$
\psi_{W}(x, z)=e^{x z}\left\{1+\frac{2}{\left(1+e^{2 x}\right) z}+\frac{1-e^{x}}{\left(1+e^{2 x}\right) z^{2}}\right\}
$$

which can be put into the form (2.13) with

$$
X=\left(\begin{array}{cc}
0 & -1 \\
1 & 0
\end{array}\right), \quad Z=\left(\begin{array}{ll}
0 & 1 \\
0 & 0
\end{array}\right),
$$

which forms a trigonometric Calogero-Moser pair as defined in (1.9), with a non-diagonalizable $Z$. The corresponding tau function is

$$
\tau_{W}\left(x, t_{2}, t_{3}, \ldots\right)=e^{-2 x}\left\{\exp \left(2 \sum_{k=2}^{\infty}(-1)^{k} t_{k}\right)-\exp \left(\sum_{k=2}^{\infty}(-1)^{k} t_{k}\right)\left(\sum_{k=2}^{\infty}(-1)^{k+1} k t_{k}\right) e^{x}+e^{2 x}\right\} .
$$

\section{$3 \mathrm{Gr}^{\text {trig }}$ and the Calogero-Moser-Sutherland hierarchy}

In this section, we relate the Grassmannian Gr $^{\text {trig }}$ introduced in Definition 1 with the CalogeroMoser-Sutherland system, also referred as the trigonometric (or hyperbolic) Calogero-Moser system. This is a system of $N$ particles on the line whose motion is governed by the Hamiltonian

$$
H(x, y)=\frac{1}{2} \sum_{i=1}^{N} y_{i}^{2}-\sum_{1 \leq i<j \leq N} \frac{1}{4 \sinh ^{2}\left((1 / 2)\left(x_{i}-x_{j}\right)\right)} .
$$

We allow the particles to move in the complex plane. The original Moser-Sutherland system [12] is recovered if we suppose our particles to be confined to the imaginary axis, since the hyperbolic sine becomes then the trigonometric one. When the motion of the particles is confined to the real axis, the potential is attractive if velocities are real, and repulsive if velocities (and time) are purely imaginary. In this last case, the particles ultimately behave like free particles. But for most initial conditions in the complex plane, some collisions will take place after a finite time.

Moser [12] proved that the system (3.1) is completely integrable, showing that it describes an isospectral deformation of the $N \times N$ matrix $L(x, y)$ with entries

$$
L_{i j}(x, y)=\delta_{i j} y_{i}+\left(1-\delta_{i j}\right) \frac{1}{2 \sinh \left((1 / 2)\left(x_{i}-x_{j}\right)\right)},
$$

where $\delta_{i j}$ is the usual Kronecker symbol. More precisely, the quantities $F_{k}(x, y)=$ $(1 / k) \operatorname{tr} L^{k}(x, y)$ (with tr denoting the trace), $k=1,2, \ldots, N$, are $N$ independent first integrals in involution for the system. In particular, $F_{2}(x, y)$ gives back the original Hamiltonian $H(x, y)$.

In order to relate the system to the KP trigonometric solitons introduced in Definition 1, we need the following lemma which can be extracted from Ruijsenaars [16], and was motivated by his study of the scattering theory of the system (3.1) when the interaction between the particles is repulsive (which, with our conventions, amounts to pick velocities and time imaginary). 
Lemma 1. Let $(X, Z) \in C_{N}^{\text {trig }}, N \geq 1$, as defined in (1.9). If $X$ is diagonalizable, there is a conjugation

$$
U^{-1} X U=K^{2}(x), \quad U^{-1} Z U=L(x, y),
$$

with $K(x)$ a diagonal matrix of the form

$$
K(x)=\operatorname{diag}\left(e^{x_{1} / 2}, \ldots, e^{x_{N} / 2}\right),
$$

and $L(x, y)$ as in $(3.2)$.

Proof. Since $\operatorname{det}(X) \neq 0$, when diagonalizing $X$, we can always assume $K$ to have the form (3.4). Denoting by $L$ the result of the conjugation of $Z$ by the same matrix $U$, since by the definition of $C_{N}^{\text {trig }}$ the rank of $[X, Z]+X$ is 1 , we have

$$
\left[K^{2}, L\right]=\alpha \beta^{t}-K^{2},
$$

with $\alpha$ and $\beta$ two (non-zero) column vectors of length $N$. Writing (3.5) componentwise, we get

$$
\left(e^{x_{i}}-e^{x_{j}}\right) L_{i j}=\alpha_{i} \beta_{j}-\delta_{i j} e^{x_{i}},
$$

which, by putting $i=j$, shows that $\alpha_{i} \beta_{i}=e^{x_{i}} \neq 0, \forall i$. Thus, by multiplying $U$ to the right by an appropriate diagonal matrix, we can always arrange that $\alpha_{i}=\beta_{i}=e^{x_{i} / 2}$. With this choice, one sees from (3.6) that necessarily $e^{x_{i}} \neq e^{x_{j}}, \forall i \neq j$, and

$$
L_{i j}=\frac{1}{2 \sinh \left((1 / 2)\left(x_{i}-x_{j}\right)\right)}, \quad \text { for } \quad i \neq j,
$$

while the diagonal entries $L_{i i}$ are free. Denoting $L_{i i}=y_{i}$ establishes the lemma.

The explicit integration of the system (3.1) was performed by Olshanetsky and Perelomov [13]. An interpretation in terms of Hamiltonian reduction was given by Kazhdan, Kostant and Sternberg [10], leading to the following result whose proof can be found in the nice treatise [19], by Suris. We identify $g l(N, \mathbb{C})$ with its dual via the trace form $\langle X, Y\rangle=\operatorname{tr}(X Y)$, and we define accordingly the gradient of a smooth function $\varphi: g l(N, \mathbb{C}) \rightarrow \mathbb{C}$ by $\mathrm{d} \varphi(X)(Y)=\langle\nabla \varphi(X), Y\rangle$.

Proposition 2. (See [19, Theorem 27.6] for a proof.) Let $\varphi: \operatorname{gl}(N, \mathbb{C}) \rightarrow \mathbb{C}$ be an Ad-invariant function, and let $H(x, y)=\varphi(L(x, y))$, with $L(x, y)$ as in $(3.2)$. Let $\left(x_{i}(t), y_{i}(t)\right)$ be the solution of Hamilton's equations

$$
\dot{x}_{i}=\frac{\partial H}{\partial y_{i}}, \quad \dot{y}_{i}=-\frac{\partial H}{\partial x_{i}},
$$

with initial conditions $\left(x_{0}, y_{0}\right)=\left(x_{1}(0), \ldots, x_{N}(0), y_{1}(0), \ldots, y_{N}(0)\right) \in \mathbb{C}^{2 N}$, such that $x_{i}(0) \neq$ $x_{j}(0), \forall i \neq j$. Then, the quantities $e^{x_{i}(t)}$ are the eigenvalues of the matrix

$$
K_{0}^{2} \exp \left(t \nabla \varphi\left(L_{0}\right)\right),
$$

with $K_{0}=K\left(x_{0}\right)$ as in (3.4), $L_{0}=L\left(x_{0}, y_{0}\right)$ and $\nabla \varphi$ the gradient of $\varphi$. Moreover, the matrix $V(t)$ which diagonalizes $K_{0}^{2} \exp \left(t \nabla \varphi\left(L_{0}\right)\right)$, so that

$$
K^{2}(x(t))=V(t) K_{0}^{2} \exp \left(t \nabla \varphi\left(L_{0}\right)\right) V(t)^{-1},
$$

and which is normalized by the condition

$$
V(t) K_{0} e=K(x(t)) e, \quad e=(1, \ldots, 1)^{t},
$$

is such that

$$
L(x(t), y(t))=V(t) L_{0} V(t)^{-1} .
$$


Remark 2. As explained at the beginning of this section, to describe a repulsive interaction in the trigonometric Calogero-Moser system with Hamiltonian $H(x, y)=\varphi(L(x, y)), \varphi(L)=$ $(1 / 2) \operatorname{tr} L^{2}$ as in (3.1) and (3.2), we have to pick the $x_{i}$ 's real, the $y_{i}$ 's imaginary and $t$ imaginary also. In this case, $t \nabla \varphi\left(L_{0}\right)=t L_{0}, t \in \sqrt{-1} \mathbb{R}$, is hermitian and thus $K_{0}^{2} \exp \left(t L_{0}\right)$ in (3.7) is always diagonalizable. In the general case, this matrix can become non-diagonalizable for some values of $t$, which leads to collisions in the system.

Let us now consider the following Hamiltonians $H_{k}(x, y)=\varphi_{k}(L(x, y))$, with

$$
\varphi_{k}(L(x, y))=\frac{1}{k+1} \operatorname{tr}\left\{(L(x, y)-I)^{k+1}-L^{k+1}(x, y)\right\}, \quad k=1,2, \ldots,
$$

and let us denote by $x_{i}(t) \equiv x_{i}\left(t_{1}, t_{2}, t_{3}, \ldots\right)$ the solution obtained by flowing along the Hamiltonian vector field $X_{H_{1}}$ during a time $t_{1}, X_{H_{2}}$ during a time $t_{2}$ etc., starting from some initial condition $\left(x_{0}, y_{0}\right) \in \mathbb{C}^{2 N}$. For short, we shall refer to $x_{i}(t) \equiv x_{i}\left(t_{1}, t_{2}, t_{3}, \ldots\right)$ as the solution of the trigonometric Calogero-Moser hierarchy with initial condition $\left(x_{0}, y_{0}\right) \in \mathbb{C}^{2 N}$.

Theorem 1. The tau function $\tau_{(X, Z-I, Z)}\left(x+t_{1}, t_{2}, t_{3}, \ldots\right)$ as given in $(2.16)$, with $(X, Z) \in C_{N}^{\text {trig }}$ and $X$ diagonalizable, is (up to an inessential exponential factor) a trigonometric polynomial in the variable $x$

$$
\tau_{(X, Z-I, Z)}\left(x+t_{1}, t_{2}, t_{3}, \ldots\right)=\prod_{i=1}^{N} 2 \sinh \frac{\left(x-x_{i}\left(t_{1}, t_{2}, t_{3}, \ldots\right)\right)}{2},
$$

where $x_{i}\left(t_{1}, t_{2}, t_{3}, \ldots\right)$ denotes the solution of the trigonometric Calogero-Moser hierarchy with initial condition $\left(x_{0}, y_{0}\right) \in \mathbb{C}^{2 N}$ specified by (3.3) as in Lemma 1 above.

Proof. Let us consider the solution $x_{i}(t)$ of the trigonometric Calogero-Moser hierarchy with initial condition $\left(x_{0}, y_{0}\right) \in \mathbb{C}^{2 N}$ specified by (3.3), i.e. $U^{-1} X U=K^{2}\left(x_{0}\right) \equiv K_{0}^{2}, U^{-1} Z U=$ $L\left(x_{0}, y_{0}\right) \equiv L_{0}$ (remember from the proof of Lemma 1 that necessarily $\left.x_{i}(0) \neq x_{j}(0), \forall i \neq j\right)$. From Proposition 2 and the definition of $\varphi_{k}$ in (3.8), it follows easily that the quantities $e^{x_{i}(t)}$ are the eigenvalues of the matrix

$$
K_{0}^{2} \exp \left\{\sum_{k=1}^{\infty} t_{k} \nabla \varphi_{k}\left(L_{0}\right)\right\}=K_{0}^{2} \exp \left\{\sum_{k=1}^{\infty} t_{k}\left(\left(L_{0}-I\right)^{k}-L_{0}^{k}\right)\right\} .
$$

From this, we deduce

$$
\begin{aligned}
& \prod_{i=1}^{N} e^{\frac{x+x_{i}(t)}{2}} 2 \sinh \frac{\left(x-x_{i}(t)\right)}{2}=\prod_{i=1}^{N}\left(e^{x}-e^{x_{i}(t)}\right) \\
& =\operatorname{det}\left\{e^{x} I-K_{0}^{2} \exp \left\{\sum_{k=1}^{\infty} t_{k}\left(\left(L_{0}-I\right)^{k}-L_{0}^{k}\right)\right\}\right\} \\
& \quad=e^{N x} \operatorname{det}\left\{I-K_{0}^{2} \exp \left\{-\left(t_{1}+x\right) I+\sum_{k=2}^{\infty} t_{k}\left(\left(L_{0}-I\right)^{k}-L_{0}^{k}\right)\right\}\right\} .
\end{aligned}
$$

Since the determinant of a matrix is invariant under conjugation, we obtain

$$
\prod_{i=1}^{N} e^{\frac{x_{i}(t)-x}{2}} \prod_{i=1}^{N} 2 \sinh \frac{\left(x-x_{i}(t)\right)}{2}=\operatorname{det}\left\{I-X \exp \left\{-\left(t_{1}+x\right) I+\sum_{k=2}^{\infty} t_{k}\left((Z-I)^{k}-Z^{k}\right)\right\}\right\} .
$$

With account of (2.16), this establishes (3.9), up to the inessential (in the sense that it leads to the same solution of the KP hierarchy) exponential factor $e^{\sum_{i=1}^{N} \frac{x_{i}(t)-x}{2}}$. The proof is complete. 


\section{The bispectral property of the KP trigonometric solitons}

The adelic Grassmannian $\mathrm{Gr}^{\mathrm{ad}}$ consists of the spaces $W \in \mathrm{Gr}^{\mathrm{rat}}$ for which the curve $\operatorname{Spec}\left(A_{W}\right)$ (with $A_{W}$ as in (2.2)) is unicursal, that is the birational isomorphism $\mathbb{C} \rightarrow \operatorname{Spec}\left(A_{W}\right)$ corresponding to the inclusion $A_{W} \subset \mathbb{C}[z]$ is bijective. As established in [20], $\mathrm{Gr}^{\text {ad }}$ parametrizes all commutative rank 1 algebras $\mathcal{A}$ of bispectral differential operators, in the sense of (1.2) and (1.3). The corresponding algebras $\mathcal{A}$ are isomorphic to $A_{W}$. The joint eigenfunction of the operators in $\mathcal{A}$ is given by the stationary Baker-Akhiezer function $\psi_{W}(x, z)$ of $W$, and it can be written in terms of a pair of matrices $(X, Z)$ satisfying $\operatorname{rank}([X, Z]+I)=1$ as in (1.4). Since in Section 2 we have chosen in the definition of $\mathrm{Gr}$ the circle $S^{1}$ to be of radius 1 , all the eigenvalues of $Z$ (which correspond to the singular points of $\operatorname{Spec}\left(A_{W}\right)$ under the map $\mathbb{C} \rightarrow \operatorname{Spec}\left(A_{W}\right)$ ) will be inside of $S^{1}$, see [21]. We shall thus assume without loss of generality that any pair $(X, Z) \in C_{N}$ as defined in (1.1) satisfies the condition that the spectrum of $Z$ is inside the unit circle ${ }^{3}$.

Following [6], starting from any $W \in \mathrm{Gr}^{\text {ad }}$ and its corresponding tau function $\tau_{W}\left(t_{1}, t_{2}, \ldots\right)$, we build a function $\psi(n, t, z)=\psi\left(n, t_{1}, t_{2}, \ldots, z\right)$ via the formula

$$
\psi(n, t, z)=(1+z)^{n} \exp (t, z) \frac{\tau_{W}\left(t_{1}+n-1 / z, t_{2}-n / 2-1 /\left(2 z^{2}\right), t_{3}+n / 3-1 /\left(3 z^{3}\right), \ldots\right)}{\tau_{W}\left(t_{1}+n, t_{2}-n / 2, t_{3}+n / 3, \ldots\right)} .
$$

We define a corresponding flag of subspaces in $L^{2}\left(S^{1}, \mathbb{C}\right)$

$$
\mathcal{V}: \cdots \subset V_{n+1} \subset V_{n} \subset V_{n-1} \subset \cdots
$$

with $V_{n}$ the closure in $L^{2}\left(S^{1}, \mathbb{C}\right)$ of the space

$$
V_{n}^{\text {alg }}=\operatorname{span} \text { of }\{\psi(n, 0, z), \psi(n+1,0, z), \psi(n+2,0, z), \ldots\} .
$$

The set of these flags was called the adelic flag manifold in [6], and we shall denote it by $\mathrm{Fl}^{\text {ad }}$. In order to formulate the main result of [6], we need to introduce the following algebra

$$
\begin{aligned}
A_{\mathcal{V}}= & \{\text { rational functions } f(z) \text { with poles only at } z=-1 \text { and } z=\infty, \\
& \text { such that } \left.\exists k \in \mathbb{Z}, \text { for which } f(z) . V_{n} \subset V_{n+k}, \forall n\right\} .
\end{aligned}
$$

It can be shown that the curve $\operatorname{Spec}\left(A_{\mathcal{V}}\right)$ is also unicursal, and that there is a bijective birational isomorphism $\mathbb{C} \backslash\{-1\} \rightarrow \operatorname{Spec}\left(A_{\mathcal{V}}\right)$ which sends -1 and $\infty$ to two smooth points completing the curve (see [6, Theorem 4.4]).

Theorem 2. (see Haine-Iliev [6]). Any $\mathcal{V} \in \mathrm{Fl}^{\text {ad }}$ gives rise to a rank one bispectral commutative algebra of difference operators $\mathcal{A}$ as in (1.6) and (1.7), isomorphic to $A_{\mathcal{V}}$ as defined in (4.2).

The function $\psi \mathcal{V}(n, z) \equiv \psi(n, 0, z)$ is the joint eigenfuntion of the operators in $\mathcal{A}$, and is called the (stationary) Baker-Akhiezer function of the flag. From the definition of $\mathrm{Fl}^{\text {ad }}$ and the result of [21] which establishes a bijection between the union of the Calogero-Moser spaces $C_{N}, N \geq 0$, introduced in (1.1) and $\mathrm{Gr}^{\text {ad }}$, it is easy to deduce the following lemma.

Lemma 2. There is a bijection $\beta: \cup_{N \geq 0} C_{N} \rightarrow \mathrm{Fl}^{\text {ad }}$ given by the map

$$
(X, Z) \rightarrow \psi_{\mathcal{V}}(n, z)=(1+z)^{n} \operatorname{det}\left\{I+\left(X-n(I+Z)^{-1}\right)^{-1}(z I-Z)^{-1}\right\} .
$$

\footnotetext{
${ }^{3}$ In [20, 21], the radius of $S^{1}$ is allowed to vary in the definition of $\mathrm{Gr}^{\text {ad }}$. As explained in [18], there is no loss of generality in fixing the radius to be 1 , since the scaling transformations $\psi_{R_{\lambda} W}(x, z)=\psi_{W}\left(\lambda x, \lambda^{-1} z\right), 0<|\lambda| \leq 1$, act on Gr as defined in Section 2.
} 
Proof. From Sato's formula (2.1) and formula (1.4) for the (stationary) Baker-Akhiezer function of a space $W \in \mathrm{Gr}^{\text {ad }}$, one deduces easily that the corresponding tau function is given by

$$
\tau_{W}\left(t_{1}, t_{2}, t_{3}, \ldots\right)=\operatorname{det}\left\{X-\sum_{k=1}^{\infty} k t_{k} Z^{k-1}\right\} .
$$

Using the fact that the spectrum of $Z$ is inside the unit circle, one finds

$$
\tau_{\mathcal{V}}\left(n, t_{1}, t_{2}, \ldots\right) \equiv \tau_{W}\left(t_{1}+n, t_{2}-\frac{n}{2}, t_{3}+\frac{n}{3}, \ldots\right)=\operatorname{det}\left\{X-\sum_{k=1}^{\infty} k t_{k} Z^{k-1}-n(I+Z)^{-1}\right\} .
$$

From (4.1), a simple computation shows then that the (stationary) Baker-Akhiezer function $\psi_{\mathcal{V}}(n, z)$ of the corresponding adelic flag is given by (4.3), which establishes the lemma.

We can now formulate the main result of our paper.

Theorem 3. The stationary Baker-Akhiezer function $\psi_{W}(x, z)$ of a space $W \in \mathrm{Gr}^{\text {trig }}$ as defined in (2.17), besides being an eigenfunction of a commutative algebra of differential operators in $x$

$$
A_{f} \psi_{W}(x, z) \equiv \sum_{\text {finitely many } j \in \mathbb{N}} a_{j}(x) \frac{\partial^{j}}{\partial x^{j}} \psi_{W}(x, z)=f(z) \psi_{W}(x, z) \quad \forall f \in A_{W},
$$

is also an eigenfunction of a commutative algebra of difference operators in the spectral variable $z$ built from an adelic flag $\mathcal{V}$, i.e.

$$
B_{g} \psi_{W}(x, z) \equiv \sum_{\text {finitely many } j \in \mathbb{Z}} b_{j}(z) \psi_{W}(x, z+j)=g(x) \psi_{W}(x, z) \quad \forall g \in A_{\mathcal{V}} .
$$

Proof. Any stationary Baker-Akhiezer function associated with a space $W$ in the Segal-Wilson Grassmannian, for which $\operatorname{Spec}\left(A_{W}\right)$ is an irreducible affine algebraic curve (which completes by adding one non-singular point at infinity), satisfies (4.5) for a commutative algebra of differential operators $A_{f}, f \in A_{W}$. This is a reformulation of the classical Burchnall-Chaundy-Krichever theory as explained in Section 6 of [18]. In particular, it applies to any $W \in \mathrm{Gr}^{\text {trig }} \subset \mathrm{Gr}^{\text {rat }}$.

From the definition of $\mathrm{Gr}^{\text {trig }}(2.17)$, the same proof as in Proposition 1 shows that the stationary Baker-Akhiezer function $\psi_{W}(x, z)$ of $W \in \mathrm{Gr}^{\text {trig }}$ is given by (2.13). Let us define

$$
\begin{aligned}
\psi^{b}(n, z) & =\psi_{W}(\log (1+z), n) \\
& =(1+z)^{n} \operatorname{det}\left\{I-X((1+z) I-X)^{-1}(n I-Z)^{-1}\right\} .
\end{aligned}
$$

We claim that $\psi^{b}(n, z)$ is the stationary Baker-Akhiezer function of an adelic flag. In view of the characterization given in (4.3) of the stationary Baker-Akhiezer function of an adelic flag, using the fact that the determinants of a matrix and its transpose are equal, it is enough to find two matrices $\tilde{X}$ and $\tilde{Z}$ satisfying $\operatorname{rank}([\tilde{Z}, \tilde{X}]+I)=1$, so that $\psi^{b}(n, z)$ can be put into the form

$$
\psi^{b}(n, z)=(1+z)^{n} \operatorname{det}\left\{I+(z I-\tilde{Z})^{-1}\left(\tilde{X}-n(I+\tilde{Z})^{-1}\right)^{-1}\right\} .
$$

It is easy to see that (4.8) agrees with (4.9) by picking

$$
\tilde{X}=Z X^{-1} \quad \text { and } \quad \tilde{Z}=X-I .
$$

Since

$$
[\tilde{Z}, \tilde{X}]+I=\left[X, Z X^{-1}\right]+I=X Z X^{-1}-Z+I,
$$

by the definition $(1.9)$ of $C_{N}^{\text {trig }}$, we have that $\operatorname{rank}([\tilde{Z}, \tilde{X}]+I)=1$, which establishes our claim. 
Since $\psi^{b}(n, z)$ is the stationary Baker-Akhiezer function of an adelic flag $\mathcal{V}$, by Theorem 2, there exists a commutative algebra of difference operators $\left\{B_{g}, g \in A_{\mathcal{V}}\right\}$, so that

$$
B_{g} \psi^{b}(n, z) \equiv \sum_{\text {finitely many } j \in \mathbb{Z}} b_{j}(n) \psi^{b}(n+j, z)=g(z) \psi^{b}(n, z) .
$$

Remembering the definition (4.7) of $\psi^{b}(n, z)$ in terms of $\psi_{W}(x, z)$, we obtain

$$
\sum_{\text {finitely many } j \in \mathbb{Z}} b_{j}(z) \psi_{W}(x, z+j)=g\left(e^{x}-1\right) \psi_{W}(x, z) \quad \forall g \in A_{\mathcal{V}}
$$

which establishes (4.6) and completes the proof of the theorem.

Corollary 1. The diagram (1.8) with $b$ and $b^{C}$ defined as in (1.11) and (1.12), $\beta^{\text {trig }}$ and $\beta$ defined respectively as in (1.13) and (4.3), is commutative.

Proof. Looking at the proof of Theorem 3 and more specifically at equations (4.7), (4.10) and (4.11), we have in fact constructed a map, the inverse of the map $b^{C}$ in (1.12)

$$
\left(b^{C}\right)^{-1}(X, Z)=\left(\left(X^{-1}\right)^{t} Z^{t}, X^{t}-I\right),
$$

with the property that

$$
\psi_{\beta^{\operatorname{trig}(X, Z)}}(x, z)=\psi_{\beta\left(\left(b^{C}\right)^{-1}(X, Z)\right)}\left(z, e^{x}-1\right)=\psi_{b\left(\beta\left(\left(b^{C}\right)^{-1}(X, Z)\right)\right)}(x, z) .
$$

This establishes the commutativity of the diagram (1.8).

Remark 3. In [8] (see also [14] for KdV solitons), it was shown that for any $W \in \mathrm{Gr}^{\mathrm{rat}}$, the Baker-Akhiezer function $\psi_{W}(x, z)$, besides satisfying (4.5), also satisfies

$$
\sum_{j=1}^{M} b_{j}(z, \partial / \partial z) \psi_{W}\left(x, z+\lambda_{j}\right)=g(x) \psi_{W}(x, z)
$$

for appropriate $\lambda_{j} \in \mathbb{C}$, with $b_{j}(z, \partial / \partial z)$ ordinary differential operators in $z$. As shown in Theorem 3, the KP trigonometric solitons are distinguished by the property that this equation can be taken to be a difference equation in $z$, with the $b_{j}$ 's functions of $z$ and the $\lambda_{j}$ 's integers.

\section{Ruijsenaars' duality revisited}

A simple computation, using the commutativity of the diagram (1.8), the definition of $\left(b^{C}\right)^{-1}$ in (4.12) and formula (4.4) for the tau function of an adelic flag, shows that for $(X, Z) \in C_{N}^{\text {trig }}$

$$
\tau_{b^{-1}\left(\beta^{\operatorname{trig}}(X, Z)\right)}\left(n, t_{1}, t_{2}, \ldots\right)=(-1)^{N}(\operatorname{det} X)^{-1} \operatorname{det}\left\{n I-Z+\sum_{k=1}^{\infty} k t_{k}(X-I)^{k-1} X\right\} .
$$

To make contact with the rational Ruijsenaars-Schneider system, we assume that $Z$ is diagonalizable, with no two eigenvalues differing by a unit. Then, by an argument similar to the one used in Lemma 1, there is a conjugation

$$
T^{-1} Z T=K^{\mathrm{RS}}(\lambda), \quad T^{-1} X T=L^{\mathrm{RS}}(\lambda, \theta),
$$

which diagonalizes $Z$ in such a way that

$$
K_{i j}^{\mathrm{RS}}=\lambda_{i} \delta_{i j}, \quad \text { with } \lambda_{i} \neq \lambda_{j}, \forall i \neq j, \quad \text { and } \quad L_{i j}^{\mathrm{RS}}=\frac{\mu_{i} \mu_{j}}{1+\lambda_{j}-\lambda_{i}}, \quad \text { with } \mu_{i} \neq 0 .
$$


Putting

$$
\mu_{i}=e^{\theta_{i} / 2} \prod_{k \neq i}\left[1-\frac{1}{\left(\lambda_{i}-\lambda_{k}\right)^{2}}\right]^{1 / 4}
$$

the rational Ruijsenaars-Schneider system [15] is the Hamiltonian system

$$
\dot{\lambda}_{i}=\frac{\partial H}{\partial \theta_{i}}, \quad \dot{\theta}_{i}=-\frac{\partial H}{\partial \lambda_{i}},
$$

with Hamiltonian $H(\lambda, \theta)=\operatorname{tr} L^{\mathrm{RS}}(\lambda, \theta)$. It is a completely integrable system, whose solution (as well as the solution of the flows commuting with it) is described by the next proposition.

Proposition 3. ([15], see also [19, Theorem 27.5] for a proof). Let $\varphi: g l(N, \mathbb{C}) \rightarrow \mathbb{C}$ be an Ad-invariant function. Then, the solution of the system (5.5) with Hamiltonian $H(\lambda, \theta)=$ $\varphi\left(L^{\mathrm{RS}}(\lambda, \theta)\right)$ and initial conditions $\left(\lambda_{0}, \theta_{0}\right)=\left(\lambda_{1}(0), \ldots, \lambda_{N}(0), \theta_{1}(0), \ldots, \theta_{N}(0)\right) \in \mathbb{C}^{2 N}$ satisfying $\lambda_{i}(0)-\lambda_{j}(0) \notin\{0,1\}, \forall i \neq j$, is such that the functions $\lambda_{i}(t)$ are the eigenvalues of the matrix

$$
K_{0}^{\mathrm{RS}}+t L_{0}^{\mathrm{RS}} \nabla \varphi\left(L_{0}^{\mathrm{RS}}\right),
$$

with $K_{0}^{\mathrm{RS}}=K^{\mathrm{RS}}\left(\lambda_{0}\right), L_{0}^{\mathrm{RS}}=L^{\mathrm{RS}}\left(\lambda_{0}, \theta_{0}\right)$ as in (5.3) and (5.4), and $\nabla \varphi$ the gradient of $\varphi$.

Let us now consider the following commuting Hamiltonians $H_{k}(\lambda, \theta)=\varphi_{k}\left(L^{\mathrm{RS}}(\lambda, \theta)\right)$, with

$$
\varphi_{k}\left(L^{R S}(\lambda, \theta)\right)=-\operatorname{tr}\left\{L^{\mathrm{RS}}(\lambda, \theta)-I\right\}^{k}, \quad k=1,2, \ldots,
$$

and define accordingly $\lambda_{i}\left(t_{1}, t_{2}, \ldots\right)$ to be the solution obtained by flowing along these various Hamiltonian vector fields, starting from some initial condition $\left(\lambda_{0}, \theta_{0}\right) \in \mathbb{C}^{2 N}$ as in Proposition 3. As before, we refer to $\lambda_{i}\left(t_{1}, t_{2}, \ldots\right)$ as the solution of the rational Ruijsenaars-Schneider hierarchy with initial condition $\left(\lambda_{0}, \theta_{0}\right) \in \mathbb{C}^{2 N}$. Our final theorem, when combined with Theorem 1 , reveals a duality between the trigonometric Calogero-Moser hierarchy and the rational RuijsenaarsSchneider hierarchy, in terms of the bispectral map $b$ defined in (1.11). This duality was first discovered by Ruijsenaars in [16], by studying the scattering theory of these systems.

Theorem 4. Let $W=\beta^{\text {trig }}(X, Z) \in \mathrm{Gr}^{\text {trig }}$, with $(X, Z) \in C_{N}^{\text {trig }}$ a trigonometric Calogero-Moser pair such that $Z$ is diagonalizable with no two eigenvalues differing by a unit. Then

$$
\tau_{b^{-1}(W)}\left(n, t_{1}, t_{2}, \ldots\right)=(-1)^{N} e^{-\sum_{i=1}^{N} \theta_{i}(0)} \prod_{i=1}^{N}\left(n-\lambda_{i}\left(t_{1}, t_{2}, \ldots\right)\right),
$$

where $\lambda_{i}\left(t_{1}, t_{2}, \ldots\right)$ is the solution of the rational Ruijsenaars-Schneider hierarchy, with initial condition $\left(\lambda_{0}, \theta_{0}\right)$ defined by (5.2), (5.3) and (5.4).

Proof. Let $L^{\mathrm{RS}}\left(\lambda_{0}, \theta_{0}\right) \equiv L_{0}^{\mathrm{RS}}$ and $K^{\mathrm{RS}}\left(\lambda_{0}\right) \equiv K_{0}^{\mathrm{RS}}$ be defined from $X$ and $Z$ as in (5.2), (5.3) and (5.4). Since a determinant is invariant under conjugation, using the definition of $\varphi_{k}(5.6)$, from (5.1) we find

$$
\tau_{b^{-1}(W)}\left(n, t_{1}, t_{2}, \ldots\right)=(-1)^{N}\left(\operatorname{det} L_{0}^{\mathrm{RS}}\right)^{-1} \operatorname{det}\left\{n I-K_{0}^{\mathrm{RS}}-\sum_{k=1}^{\infty} t_{k} L_{0}^{\mathrm{RS}} \nabla \varphi_{k}\left(L_{0}^{\mathrm{RS}}\right)\right\} .
$$

By Cauchy's determinant formula (2.14), using (5.4), we get

$$
\left(\operatorname{det} L_{0}^{\mathrm{RS}}\right)^{-1}=\prod_{i=1}^{N} e^{-\theta_{i}(0)}
$$


From Proposition 3, it follows that the functions $\lambda_{i}\left(t_{1}, t_{2}, \ldots\right)$ which solve the rational Ruijsenaars-Schneider hierarchy with initial condition $\left(\lambda_{0}, \theta_{0}\right)$ are the eigenvalues of the matrix

$$
K_{0}^{\mathrm{RS}}+\sum_{k=1}^{\infty} t_{k} L_{0}^{\mathrm{RS}} \nabla \varphi_{k}\left(L_{0}^{\mathrm{RS}}\right),
$$

which establishes the assertion (5.7) and completes the proof of the theorem.

Remark 4. A trigonometric Calogero-Moser pair $(X, Z)$ for which both $X$ and $Z$ are diagonalizable with no two eigenvalues of $Z$ differing by a unit, can be represented (modulo conjugation) by $\left(K^{2}\left(x_{0}\right), L\left(x_{0}, y_{0}\right)\right)$ as in $(3.3)$, or $\left(L^{\mathrm{RS}}\left(\lambda_{0}, \theta_{0}\right), K^{\mathrm{RS}}\left(\lambda_{0}\right)\right)$ as in (5.2). Let $W=\beta^{\text {trig }}(X, Z)$ be the corresponding space in $\mathrm{Gr}^{\text {trig }}$. By Theorem 1 , the zeros of $\tau_{W}\left(x+t_{1}, t_{2}, \ldots\right)$ as a function of $x$ solve the trigonometric Calogero-Moser hierarchy with initial condition $\left(x_{0}, y_{0}\right)$ and, by Theorem 4, the zeros as a function of $n$ of $\tau_{b^{-1}(W)}\left(n, t_{1}, t_{2}, \ldots\right)$ solve the rational RuijsenaarsSchneider hierarchy with initial condition $\left(\lambda_{0}, \theta_{0}\right)$. To describe a repulsive interaction in the trigonometric Calogero-Moser system (3.1), one chooses the $x_{i}$ 's real with $x_{1}<\cdots<x_{N}$, the $y_{i}$ 's imaginary and time imaginary too. In this case $L(x, y)$ is anti-hermitian and thus diagonalizable with imaginary eigenvalues. As shown in [16, Section $2 \mathrm{C}]$, the matrix $T$ in (5.2) which conjugates the pair $\left(K^{2}(x), L(x, y)\right)$ to $\left(L^{\mathrm{RS}}(\lambda, \theta), K^{\mathrm{RS}}(\lambda)\right)$, is then uniquely determined by requiring it to be unitary and such that $\sqrt{-1} \lambda_{1}<\cdots<\sqrt{-1} \lambda_{N}$ and $\mu_{i}>0$. The real solution of the hyperbolic Calogero-Moser system is then given by $\left(x_{i}(\sqrt{-1} t), \sqrt{-1} y_{i}(\sqrt{-1} t)\right), t \in \mathbb{R}$, and the map $\left(\sqrt{-1} y_{i}, x_{i}\right) \rightarrow\left(\sqrt{-1} \lambda_{i}, \theta_{i}\right)$, is the scattering map, providing the action-angle variables for the system. Vice versa, the map $\left(\theta_{i}, \sqrt{-1} \lambda_{i}\right) \rightarrow\left(x_{i}, \sqrt{-1} y_{i}\right)$ gives the action-angle variables for the rational Ruijsenaars-Schneider system.

\section{Acknowledgements}

I wish to thank S.N.M. Ruijsenaars for his comments about [6] during the 'International Workshop on Special Functions, Orthogonal Polynomials, Quantum Groups and Related Topics' dedicated to Dick Askey 70th birthday (Bexbach, October 2003), which hinted at some of the results presented here, as well as for sending [17]. I also thank two anonymous referees for stimulating suggestions, which led to improvement of the final form of the paper. Partial support from the European Science Foundation Programme MISGAM, the Marie Curie RTN ENIGMA and a Grant of the Belgian National Science Foundation (FNRS) are also gratefully acknowledged.

\section{References}

[1] Chalykh O.A., The duality of the generalized Calogero and Ruijsenaars problems, Russian Math. Surveys 52 (1997), 1289-1291.

[2] Chalykh O.A., Bispectrality for the quantum Ruijsenaars model and its integrable deformation, J. Math. Phys. 41 (2000), 5139-5167.

[3] Duistermaat J.J., Grünbaum F.A., Differential equations in the spectral parameter, Comm. Math. Phys. 103 (1986), 177-240.

[4] Etingof P., Lectures on Calogero-Moser systems, math.QA/0606233.

[5] Grünbaum F.A., Haine L., A theorem of Bochner revisited, in Algebraic Aspects of Integrable Systems: In Memory of Irene Dorfman, Editors A.S. Fokas and I.M. Gelfand, Progr. Nonlinear Differential Equations, Vol. 26, Birkhäuser, Boston, MA, 1997, 143-172.

[6] Haine L., Iliev P., Commutative rings of difference operators and an adelic flag manifold, Int. Math. Res. Not. 6 (2000), 281-323.

[7] Iliev P., Rational Ruijsenaars-Schneider hierarchy and bispectral difference operators, math-ph/0609011. 
[8] Kasman A., Spectral difference equations satisfied by KP soliton wavefunctions, Inverse Probl. 14 (1998), 1481-1487, solv-int/9811009.

[9] Kasman A., Gekhtman M., Solitons and almost-intertwining matrices, J. Math. Phys. 42 (2001), 3540-3551, math-ph/0011011.

[10] Kazhdan D., Kostant B., Sternberg S., Hamiltonian group actions and dynamical systems of Calogero type, Comm. Pure Appl. Math. 31 (1978), 481-507.

[11] Kuznetsov V.B., Nijhoff F.W., Sklyanin E.K., Separation of variables for the Ruijsenaars system, Comm. Math. Phys. 189 (1997), 855-877, solv-int/9701004.

[12] Moser J., Three integrable systems connected with isospectral deformations, Adv. Math. 16 (1975), 197-220.

[13] Olshanetsky M.A., Perelomov A.M., Completely integrable Hamiltonian systems connected with semisimple Lie algebras, Invent. Math. 37 (1976), 93-108.

[14] Reach M., Difference equations for $N$-soliton solutions to KdV, Phys. Lett. A 129 (1988), 101-105.

[15] Ruijsenaars S.N.M., Schneider H., A new class of integrable systems and its relation to solitons, Ann. Physics 170 (1986), 370-405.

[16] Ruijsenaars S.N.M., Action-angle maps and scattering theory for some finite-dimensional integrable systems I. The pure soliton case, Comm. Math. Phys. 115 (1988), 127-165.

[17] Ruijsenaars S.N.M., Integrable particle systems vs solutions to the KP and 2D Toda equations, Ann. Physics 256 (1997), 226-301.

[18] Segal G., Wilson, G., Loop groups and equations of KdV type, Publ. Math. Inst. Hautes Études Sci. 61 (1985), 5-65.

[19] Suris Y.B., The problem of integrable discretization: Hamiltonian approach, Progr. Math. Vol. 219, Birkhäuser, Boston, MA, 2003.

[20] Wilson G., Bispectral commutative ordinary differential operators, J. Reine Angew. Math. 442 (1993), 177-204.

[21] Wilson G., Collisions of Calogero-Moser particles and an adelic Grassmannian, Invent. Math. 133 (1998), $1-41$. 